\title{
Vena subclavia ist der sicherste Ort
}

Fragestellung: Gibt es Unterschiede im Hinblick auf vaskuläre Komplikationen, wenn ein zentraler Venenkatheter in der Vena subclavia, der Vena jugularis oder der Vena femoralis angelegt wird?

Hintergrund: Viele Patienten mit schweren Erkrankungen benötigen einen zentralvenösen Zugang. Dieser kann in der Vena subclavia, Vena jugularis oder Vena femoralis gelegt werden. Zentrale Venenkatheter können Parienti JJ, Mongardon N, Mégarbane B et al; 3SITES Study Group. Intravascular complications of central venous catheterization by insertion site. $N$ Engl J Med. 2015; doi: 10.1056/NEJ-

Moa1500964 zu Infektionen, Thrombosen oder mechanischen Komplikationen führen. Bisher gab es keine große randomisierte Studie auf einer Intensivstation, die die drei Zugangswege für einen Venenkatheter untersucht hätten.
Patienten und Methodik: Die Studie schloss erwachsene Patienten ein, die auf einer Intensivstation lagen und einen venösen Zugang benötigten. Der primäre Endpunkt der Studie waren katheterbedingte Infektionen und tiefe Venenthrombosen.

Ergebnisse: Die Hälfte der Patienten erhielt eine antibiotische Therapie und $30 \%$ wurden antikoaguliert. Bei Anlage des Katheters im Bereich der A. subclavia kam es zu acht primären Ereignissen, in der Vena jugularis zu 20 und in der Vena femoralis zu 22 (Unterschied statistisch signifikant). Allerdings kam es bei Anlage des Katheters im Bereich der Vena subclavia signifikant häufiger zu einem Pneumothorax als bei der Vena jugularis (13 vs. 4$)$.

Schlussfolgerungen: Bei Patienten die einen zentralen Venenkatheter benötigen, findet sich die geringste Komplikationsrate bei Anlage des Katheters in der Vena subclavia.

\section{- Kommentar von Hans-Christoph Diener, Essen}

\section{Das Pneumothoraxrisiko sollte nicht unterschätzt werden}

Bei Patienten, die einen zentralen Venenkatheter benötigen, ist die Anlage des Katheters im Bereich der Vena subclavia mit den geringsten Komplikationsraten (außer ein Pneumothorax) assoziiert. Insgesamt hatte die Studie eine erstaunlich geringe Rate von Infektionen durch den Katheter, was daran lag, dass im Rahmen der Studie klare Instruktionen bezüglich Katheter- anlage und Desinfektion gegeben wurden. Die Anlage des Katheters im Bereich der Vena subclavia wurde auch häufiger ultraschallgestützt durchgeführt.

Allerdings sollte das Risiko eines Pneumothorax bei der Anlage des Katheters in der Vena subclavia nicht unterschätzt werden.

\section{Können autistische Kernsymptome doch trainiert werden?}

Fragestellung: Die Studie von Sven Bölte et al. vom Karolinska Institut in Schweden untersucht die Fähigkeit von autistischen Menschen, den affektiven Gehalt von Gesichtern zu erkennen (facial affect recognition, FAR) verglichen mit den Leistungen einer Kontrollgruppe. Mithilfe der funktionellen Bildgebung wurde analysiert, welche zerebralen Netzwerke dabei beteiligt sind. In einem zweiten Längsschnittteil wurde geschaut, ob eventuelle Defizite der FAR durch computerbasierte Trainingsprogramme verbessert werden können und wie sich dies auf die Neurobiologie auswirkt.

Hintergrund: Autismus-Spektrum-Störungen (ASS) werden in den letzten Jahren zunehmend als eine wichtige Differenzial- diagnose auch in der Erwachsenenpsychiatrie und Psychotherapie erkannt. Denn nicht selten verbirgt sich ein hochfunktionaler Autismus als eine für Arzt wie Patient plausible und authentische Erklärung hinter einem komplexen und von vielen Konflikten gesäumten Lebensweg, mit vielen unterschiedlichen psychiatrischen Vordiagnosen wie Persönlichkeitsstörungen (vor allem Borderline-Störung bei Frauen und narzisstische oder kombinierte Persönlichkeitsstörung bei Männern), Depressionen, atypischen Zwangsstörungen, Essstörungen, Anpassungsstörungen etc. $[1,2]$. Neben der zentralen gestörten sozialen Kognition in Form einer nur bedingt möglichen Perspektivübernahme und intuitiven Theory-of-Mind-Fähigkeit (synonym: kognitive Empathie oder Mentalisierungsfähigkeit) 